\title{
Guiding group cognition in CSCL
}

\author{
Gerry Stahl
}

Published online: 30 June 2010

(C) International Society of the Learning Sciences, Inc.; Springer Science+Business Media, LLC 2010

Regardless of whether particular stakeholders are interested in individual learning outcomes or in the knowledge-building accomplishments of teams, the power of collaborative learning emanates from its potential to coalesce multiple people into the coherent cognitive effort of a group. The research goal of the field of CSCL is to understand how this synergy takes place and to design ways of supporting its fragile processes. The rigorous study of group cognition is elusive because successful collaborative learning is (a) currently rare and hard to identify, (b) complex in the structure of its constituent mechanisms and the factors influencing them, and (c) unique in each of its situated instances.

There are now a number of theoretical frameworks available, which are influential in the CSCL research community, each, perhaps, with its own model of the influences on collaborative learning that must be taken into account. Figure 1 is an attempt to visualize major categories of these influences. It places at the center the dialogical interaction through which individual participants form into a collective knowledge-building agency.

The sequential nature of the interaction is what weaves contributions from the Bakhtinian voices of individuals into group processes of meaning making, as each responds to previous entries and elicits new ones. The meanings - shared by the group by virtue of their having been co-constructed in the collectively experienced sequential interaction - are embodied in team knowledge artifacts, whether linguistic phrases or physical objects. This collaborative knowledge building produces the team's outcomes, which are driven by the team's task.

A major thrust of the CSCL research agenda is to analyze the influences and constraints on the flow of knowledge building sketched in the preceding paragraph. Of course, a starting point is the determination of the individual voices of the participants: their background, perspectives, and abilities. What experiences do they bring to the interaction and what resources can they each contribute? These factors at the individual unit of analysis are preconditions of the collaboration; they are of interest to education and psychology in general, but not specifically CSCL's concerns, which are more directed toward the group level of description.

G. Stahl $(\bowtie)$

Drexel University, Philadelphia, PA, USA

e-mail: Gerry.Stahl@drexel.edu 


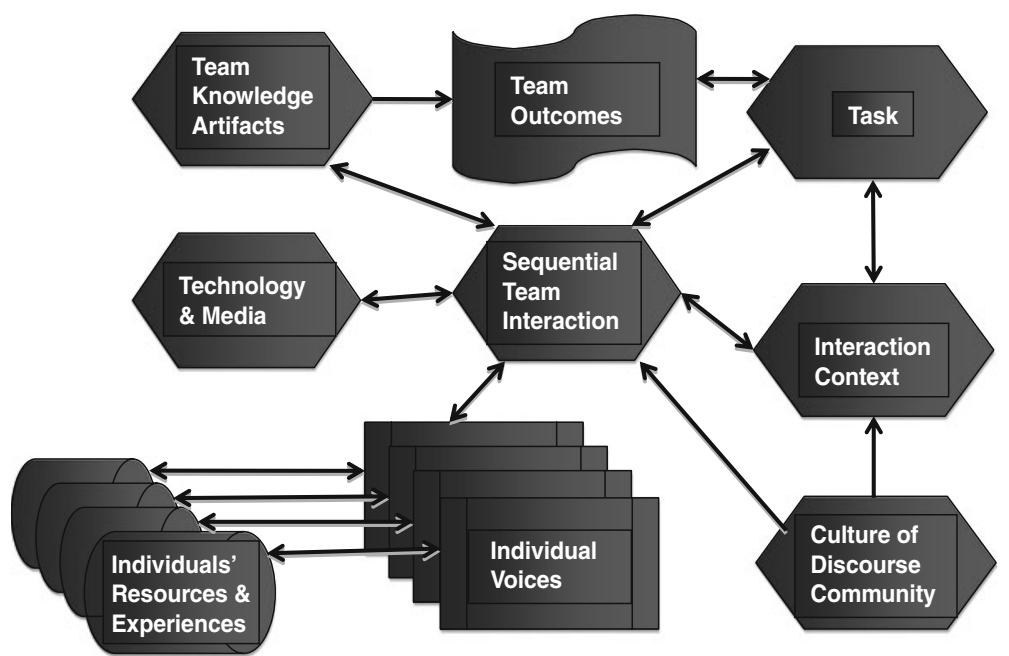

Fig. 1 A diagram of major influences on group cognition

By virtue of its name and its history, CSCL is especially oriented toward the computational technology and the digital media that support online group interaction. In addition, theories of situativity, activity, ethnomethodology, actor networks, and distributed cognition highlight the essential influences on collaboration of the ongoing interactional context, the teleological object of the activity, available conceptual tools, established social practices, immutable-mobile mediators, the evolving joint problem space, and the larger socio-cultural horizon.

Because CSCL is an empirical science, researchers must capture data that lends itself to the analysis of these various dimensions of group interaction. To plausibly demonstrate the nature of particular influences, they must somehow focus on the phenomena they wish to study and determine the role they are playing. The authors of the papers in this issue do so in very different ways, illustrating once more the vigorous diversity, which is a core strength of the CSCL research field. The first four studies investigate how various forms of scaffolding can guide the group interaction in a pedagogically desirable direction, while the final reflection shows that the interaction also depends upon - and helps to constructinternal preconditions of productive collaboration, such as mutual trust.

The opening paper by Christa S. C. Asterhan and Baruch B. Schwarz starts with a useful literature review of the most basic form of scaffolding: that in which an instructor personally intervenes to guide synchronous small-group discussions. The paper then looks at four classes that are using an online environment to structure argumentation while a teacher is participating with each small group as a moderator, using various typical styles of facilitation. First, student self-reports from the students are compiled about what form of moderation seemed most effective to them, and then knowledge-building artifacts from the classes are analyzed to determine the effectiveness of the teacher intervention. Underlining the ways in which different factors interact with each other (and thereby complicating the task of modeling the dimensions of collaboration as though they were independent factors), the authors stress how different the moderation of synchronous computer-mediated interaction is from that of face-to-face or asynchronous interaction. Furthermore, they 
report that different approaches to moderation taken by different teachers exhibit very different characteristics and results.

The next contribution to this issue reviews the concept of scaffolding further and explores it in the context of medical-school training. Problem-based learning (PBL) has been a popular form of small-group collaborative learning in medical schools for decades. Jingyan Lu, Susanne P. Lajoie, and Jeffrey Wiseman have been exploring ways to extend the PBL model to overcome certain of its limitations. Here they report on changes to the effectiveness of teacher scaffolding due to two innovations: (a) an innovative form of medical case for role-playing called "the deteriorating patient" and (b) the use of interactive whiteboards. They analyze the changes in scaffolding strategies and discourse patterns in response to these innovations.

The contributions to group discourse made by a given individual are obviously influenced by the information and knowledge that the person has - or the experiences and resources available to them. Their contributions are likely to gradually introduce this information into the group knowledge-building or problem-solving process. In fact, much of the power of collaborative learning can come from the pooling of different knowledge and alternative perspectives distributed within the group. However, finding out who knows what can take time and delay the ultimate problem solving. The experiment reported by Tanja Engelmann and Friedrich W. Hesse ${ }^{1}$ investigates how information about what the group participants each know can be introduced into the shared group understanding through the use of CSCL technology. Specifically, they use the popular classroom tool of concept maps, having each participant within the experimental condition display for their collaborators a concept map representing their own knowledge. Triads with access to each other's concept maps proved to be more efficient in their collaborative problem solving.

The traditional concept of scaffolding, going back at least to Vygotsky, involved teachers or other students supporting collaboration and learning. Within CSCL, software tools (like argumentation environments, interactive whiteboards, or concept maps) have been used to support specific educational activities, and automated scripts have been used to guide students and teams through consecutive phases of a planned learning trajectory. CSCL researchers have found that the creation of one-off scripts is time consuming and hard to scale up for widespread classroom usage. For this reason, Christof Wecker, et al. discuss their effort to develop an infrastructure for scripts that can be ported to different collaboration environments. They do this by means of a browser plug-in, which can recognize inputs from different CSCL systems and provide responses in accordance with a cross-platform script definition. They illustrate its application in a realistic educational application setting.

CSCL researchers can become focused on trying to promote and control collaboration from outside the group itself. Taken too far, this can result in the fostering and administering of strategic communication and impression management, furthering external goals at the expense of the group's own autonomy, agency, and sociability. Students can be influenced to engage in strategies designed to earn high grades rather than to build knowledge. For that reason, we close this reflection on guiding group cognition in CSCL by returning to the interpersonal resources of the group participants themselves. In the final paper of the issue, Anne Gerdes guides us in thinking about relations of trust among people:

\footnotetext{
${ }^{1}$ Friedrich Hesse was not involved in reviewing for this issue.
} 
both how trust is required by collaborative undertakings as a spontaneous embodied experience of being-in-the-world-with-others and also how it may be engendered by the collaboration process itself. In contrast to journal articles that adopt an appearance of objectivity, this essay represents a new genre for ijCSCL: that of a brief, but deep reflection piece from a pointed perspective.

ijCSCL is now the \#2 educational journal in the world. ISI Web of Knowledge just released its report that $i j C S C L$ has an impact factor of 2.692 , the second highest impact factor of the 139 ISI-indexed journals in the category "Education and Educational Research."

Thanks to all our Editorial Board members, reviewers, authors, and subscribers for your support! Thanks to the active CSCL research community, which reads and cites $i j C S C L$ ! 